\title{
A linear quadratic regulator for nonlinear SIRC epidemic model
}

\author{
Paolo Di Giamberardino \\ Dept. Computer, Control and Management \\ Engineering Antonio Ruberti \\ Sapienza University of Rome \\ Rome, Italy \\ paolo.digiamberardino@uniroma1.it
}

\author{
Daniela Iacoviello \\ Dept. Computer, Control and Management \\ Engineering Antonio Ruberti \\ Sapienza University of Rome \\ Rome, Italy \\ daniela.iacoviello@uniroma1.it
}

\begin{abstract}
The control of an epidemic disease consists in introducing the strategies able to reduce the number of infected subjects by means of medication/quarantine actions, and the number of the subjects that could catch the disease through an informative campaign and, when available, a vaccination strategy. Some diseases, like the influenza, do not guarantee immunity; therefore, the subjects could get ill again by different strain of the same viral subtype. The epidemic model adopted in this paper introduces the cross-immune individuals; it is known in literature as SIRC model, since the classes of susceptible (S), infected (I), removed (R) and cross-immune (C) subjects are considered. Its control is herein determined in the framework of the linear quadratic regulator, by applying to the original nonlinear model the optimal control found on the linearized system. The results appear satisfactory, and the drawback of using a control law based on the linear approximation of the system is compensated by the advantages arising from such a solution: no costate equations to be solved and a solution depending on the current state evolution which allows a feedback implementation.
\end{abstract}

Keywords-epidemic diseases modelling; SIRC epidemic model; optimal control; linear quadratic control

\section{INTRODUCTION}

Epidemic modelling has increased its importance in the last decades showing its power when facing with severe and dangerous diseases, [1], [2], [3], [4], [5], [6]. In the mathematical models, the population is generally divided into classes or compartments; the basic model is the SIR one, where $S$ represents the susceptible subjects, i.e. the ones that could get the disease, I stands for the infected individuals and $\mathrm{R}$ denotes the recovered subjects, i.e. the ones that, after having got the infection, are again healthy. The SIRC model, firstly introduced in [7], includes the cross-immune subjects $\mathrm{C}$, the ones that after a disease do not have the total immunization for a different strain of the same viral subtype, [3], [8]. In influenza-like epidemic diseases, subjects acquire a temporary immunity and then, with a given probability, could catch the disease again, [9], [10].

Controlling an epidemic disease means to apply actions aiming at reducing the effects of the infection and the spread of the virus; this suggests the introduction of prophylaxis

This work was supported by Sapienza University of Rome, Grants No. 643-009-18 and 2018 and treatments, like vaccination and/or information campaign, medication and quarantine, taking into account the resources limitations. The useful framework in which epidemic modelling and control are suitably studied is the optimal control. In [1] it is applied to different deterministic models with the goal of minimizing the number of infected subjects with as less resources as possible, by using the Pontryagin minimum principle. The literature on the optimal control of specific epidemic disease is vast, introducing actions specific for the considered disease, like in [11], [12], [13] and in [2], for the SIR epidemic model, or [14], [15] and [16] for the measles, or [17], [18],[19],[20], [21] for the HIV/AIDS, just to mention the most recent papers on diffused diseases. In [3], the SIRC model is considered and an optimal control strategy aiming at the reduction of susceptible and infected individuals is proposed, introducing two controls, both on susceptible (i.e. the vaccination and/or the informative campaign) and on the infected (i.e. the treatment), taking into account the limitations of the resources. Despite the solution provides acceptable results, showing a significant reduction of the number of the infected patients, it is not available in the form of a feedback law. In this paper a quadratic cost index with the same goal herein discussed is proposed; the adopted SIRC model is nonlinear for the presence of the interaction terms. The case of nonlinear dynamical system with a quadratic cost index is rather common and inspired different solutions, depending on the specific model and on the nonlinearity, [22], [23], [24]. In [24] this problem is faced referring to a musculo-skeletal arm discrete model; the authors developed an iterative linear quadratic regulator algorithm based on an iterative linearization of the nonlinear model. Nonlinear epidemic models describing attacks on computer networks are studied in [25], [26]; in particular, in [26] a linear feedback controller is designed making use of a linear approximation obtained disregarding the nonlinear terms. In this paper the nonlinear terms are not disregarded, but a linearization of the original SIRC model in a neighborhood of an equilibrium point is considered. The minimization of the quadratic cost index yields an optimal feedback law for the linearized system and the same law is applied to the original nonlinear model. The feedback control law allows to avoid the calculation 
of the solution of the costate equations and the consequent only numerical evaluation of the solution of the optimal control problem. The numerical results appear satisfactory, with comparable behaviours of the dynamics with respect to analogous optimization procedures. The paper is organized as follows; in Section II the SIRC model is briefly recalled and the proposed control strategy is outlined; in Section III some simulations are proposed to show the effectiveness of the approach, while some conclusions and future work indications are in Section IV.

\section{THE SIRC MODEL AND THE PROPOSED CONTROL ACTIONS}

The SIRC model considered includes four categories of subjects; along with the susceptible individuals $\mathrm{S}$, the infected and infectious patients $\mathrm{I}$ and the recovered people $\mathrm{R}$, the cross-immune subjects $\mathrm{C}$ are introduced. After a period, the recovered individuals move to the class $C$ : they have only partial immunity to a new dominant strain of the same virus subtype already faced. A fraction of the exposed cross immune individuals is recruited into the $\mathrm{R}$ compartment, having a positive immune response, whereas the others could get the disease again, and therefore move to the susceptible class, or directly become infected, Fig.1. In absence of cross immunity, the classes of susceptible and cross-immune subjects are immunologically indistinguishable, as shown in [7]. The model adopted is characterized by one control action on the susceptible subjects as information campaigns and/or by vaccinations, and by a second one on the infected patients, corresponding to medications and/or quarantine; it is nonlinear, due to the interactions between individuals of different compartments and it is described by the equations

$$
\begin{array}{r}
\dot{S}(t)=\mu(1-S(t))-\beta S(t) I(t)+\gamma C(t)-\rho_{1} S(t) u_{1}(t) \\
\dot{I}(t)=\beta S(t) I(t)+\sigma \beta C(t) I(t)-(\mu+\alpha) I(t) \\
-\rho_{2} I(t) u_{2}(t) \\
\dot{R}(t)=(1-\sigma) \beta C(t) I(t)+\alpha I(t)-(\mu+\delta) R(t) \\
+\rho_{1} S(t) u_{1}(t)+\rho_{2} I(t) u_{2}(t) \\
\dot{C}(t)=\delta R(t)-\beta C(t) I(t)-(\mu+\gamma) C(t)
\end{array}
$$

with initial conditions:

$$
S(0)=S_{0}, \quad I(0)=I_{0}, \quad R(0)=R_{0}, \quad C(0)=C_{0}
$$

As usually, the quantities $S, I, R, C$ are normalized and may be interpreted as percentage of population. The parameters $\alpha, \beta, \delta, \gamma, \mu, \sigma$ are all real and positive with the following meaning, [7]: $\alpha, \delta$ and $\gamma$ the inverses of the average time spent by the subjects in the compartments $I, R$ and $C$ respectively; $\beta$ is the contact rate; $\mu$ represents the mortality rate in each compartment and it is assumed equal to the rate of the newborn population; the average reinfection probability of a crossimmune subject is given by $\sigma$. The control parameters $\rho_{1}$

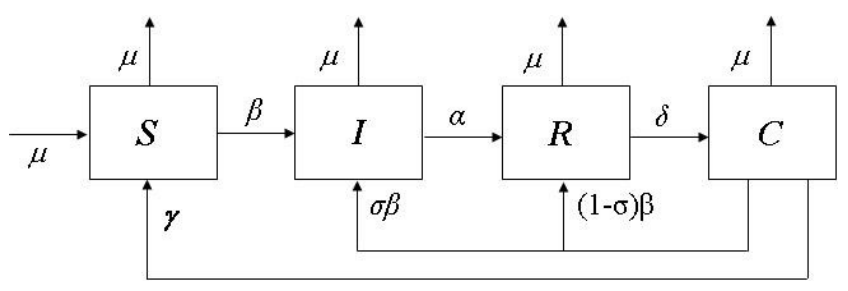

Fig. 1. Block diagram of the SIRC epidemic model without control action

and $\rho_{2}$ represent the efficiency of the control action on the evolution of susceptible and infected subjects.

The above system (1)-(4) may be rewritten in the compact form

$$
\dot{X}=F(X, U)=f(X)+g_{1}(X) u_{1}+g_{2}(X) u_{2}
$$

where

$$
\begin{aligned}
& X(t)=\left(\begin{array}{llll}
S(t) & I(t) & R(t) & C(t)
\end{array}\right)^{T} \\
& U(t)=\left(u_{1}(t) \quad u_{2}(t)\right)^{T}
\end{aligned}
$$

and

$$
\begin{gathered}
f(X)=\left(\begin{array}{c}
\mu(1-S(t))-\beta S(t) I(t)+\gamma C(t) \\
\beta S(t) I(t)+\sigma \beta C(t) I(t)-(\mu+\alpha) I(t) \\
(1-\sigma) \beta C(t) I(t)+\alpha I(t)-(\mu+\delta) R(t) \\
\delta R(t)-\beta C(t) I(t)-(\mu+\gamma) C(t)
\end{array}\right) \\
g_{1}(X)=\left(\begin{array}{c}
-\rho_{1} S(t) \\
0 \\
\rho_{1} S(t) \\
0
\end{array}\right) \\
g_{2}(X)=\left(\begin{array}{c}
0 \\
-\rho_{2} I(t) \\
\rho_{2} I(t) \\
0
\end{array}\right)
\end{gathered}
$$

The aim of this work is to determine the optimal control $U(t)$ that minimizes the susceptible and the infected individuals, as well as the control action, according to the quadratic cost function:

$$
\begin{aligned}
& J=\frac{1}{2} \int_{t_{0}}^{\infty}\left(x^{T} Q x+U^{T} R U\right) d t \\
& =\frac{1}{2} \int_{t_{0}}^{\infty}\left(q_{1} X_{1}^{2}(t)+q_{2} X_{2}^{2}(t)+r_{1} u_{1}^{2}(t)+r_{2} u_{2}^{2}(t)\right) d t
\end{aligned}
$$

The system (1)-(4) is linearized in the neighborhood of one of the equilibrium points $X_{i}^{e}, i=1,2, \ldots, n$ obtained as the solutions of the equation $F\left(X_{i}^{e}, 0\right)=0, i=1,2, \ldots, n$.

For the linearized dynamics, the following matrices must be computed:

$$
\begin{aligned}
& A_{i}=\left.\frac{\partial F}{\partial X}\right|_{\substack{X=X_{i}^{e} \\
U=0}}= \\
& \left(\begin{array}{cccc}
-\mu-\beta I & \beta S & 0 & \gamma \\
\beta I & \beta S+\sigma \beta C-(\mu+\alpha) & 0 & \sigma \beta I \\
0 & (-\sigma) \beta C+\alpha & -(\mu+\delta) & (1-\sigma) \beta I \\
0 & -\beta C & \delta & \beta I-(\mu+\gamma) C
\end{array}\right)_{\begin{array}{c}
X=X_{i}^{e} \\
U=0
\end{array}}
\end{aligned}
$$




$$
\begin{aligned}
B_{i} & =\left.\frac{\partial F}{\partial\left(u_{1}, u_{2}\right)}\right|_{\substack{X=X_{i}^{e} \\
U=0}} \\
& =\left(\begin{array}{cc}
-\rho_{1} S & 0 \\
0 & -\rho_{2} I \\
\rho_{1} S & \rho_{2} I \\
0 & 0
\end{array}\right)_{\substack{X=X_{i}^{e} \\
U=0}}
\end{aligned}
$$

for $i=1,2, \ldots, n$.

The choice of the equilibrium point depends on its value and on the controllability characteristics of the linearized system. A synthesizable control may be determined by using the linear quadratic regulator theory, which yields a state dependent (feedback) control law to be applied to the original nonlinear system. In order to minimize the number of susceptible and infected subjects, using as less resources as possible, the optimization problem can be formulated as follows: given the locally linearized system

$$
\begin{aligned}
& \dot{\tilde{X}}(t)=A_{i} \tilde{X}(t)+B_{i} U(t) \\
& \tilde{X}(0)=0
\end{aligned}
$$

where $\tilde{X}(t)=X(t)-X_{i}^{e}$, find the optimal control $U^{o}(t)$ that minimizes the quadratic cost index:

$$
\begin{array}{r}
J=\frac{1}{2} \int_{t_{0}}^{\infty}\left(\left(\tilde{X}(T)+X_{i}^{e}\right)^{T} Q\left(\tilde{X}(t)+X_{i}^{e}\right)\right. \\
\left.+U^{T}(t) R U(t)\right) d t
\end{array}
$$

Note that in this formulation no control constraint is present, thus leaving to the quadratic term $U^{T} R U$ the reasonable limitations in resources allocation. Expression (13) arises from (9) once the state variables transformation $X(t)=\tilde{X}(t)+X_{i}^{e}$ is performed; with this structure, the problem assumes the form of a LQ tracking one. It is defined on the infinite interval $[0, \infty)$ and the matrices $A_{i}, B_{i}, Q, R$ are constant; therefore, to solve the problem, the solution of the Riccati algebraic equation:

$$
0=P B_{I} R^{-1} B_{i}^{T} P-P A_{i}-A_{i}^{T} P-Q
$$

must be found. The optimal control for the problem (12)(13) is obtained in closed form:

$$
U^{o}(t)=R^{-1} B_{i}^{T}\left[g_{r}-P \tilde{X(t)}\right]
$$

where

$$
g_{r}=-\left[P B R^{-1} B^{T}-A^{T}\right]^{-1} Q X_{i}^{e}
$$

with the optimal evolution $X^{o}(t)$ solution of the equation:

$$
\begin{aligned}
\dot{\tilde{X}}^{o}(t) & =A_{i} \tilde{X}^{o}(t)+B_{i} U^{o}(t) \\
& =\left(A_{i}-B_{i} R^{-1} B_{i}^{T} P\right) \tilde{X}^{o}(t)+-B_{i} R^{-1} B_{i}^{T} g_{r} \\
& =\bar{A}_{i} \tilde{X}^{o}(t)+B_{i} R^{-1} B_{i}^{T} g_{r}
\end{aligned}
$$

with initial condition

$$
\tilde{X}\left(t_{0}\right)=0, i=1,2, \ldots, n
$$

Note that, obviously, also the solution of the Riccati equation (14) and the correction term (16) depend on the considered equilibrium point $X_{i}^{o}$. The consequent control law for the original nonlinear system

$$
U(t)=R^{-1} B_{i}^{T}\left[g_{r}-P\left(X(t)-\tilde{X}_{i}^{e}\right)\right]
$$

is obtained from (15) substituting $\tilde{X}(T)=X(t)-X_{i}^{e}$. The characteristics in terms of stability and convergence as well as the effects of the obtained optimal control on the linearized system are well known in literature; the interesting aspect to be investigated is the behaviour of the control (19) on the original nonlinear system (1)-(4), for which it constitutes a locally approximated solution. In Fig. 2 it is shown the block diagram of the proposed control scheme. In the next Section, this analysis is presented, showing the effectiveness of the proposed control through the results of numerical simulations.

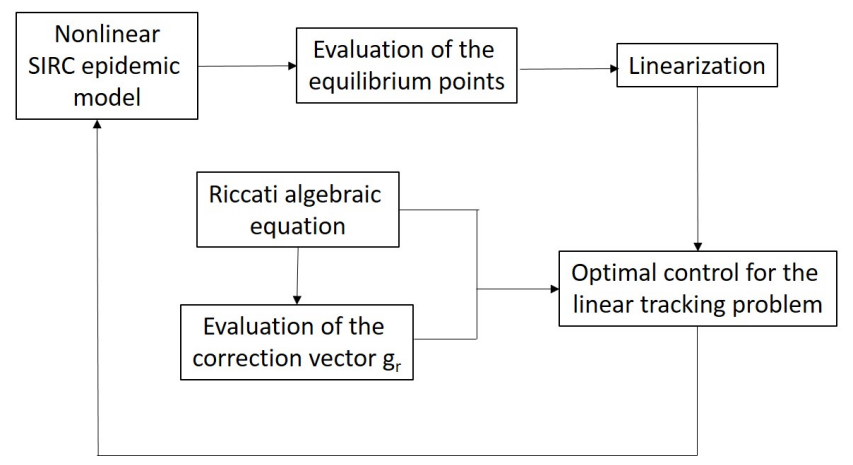

Fig. 2. Block diagram of the proposed control scheme

\section{NUMERICAL RESULTS}

The following values for the parameters are used:

$$
\begin{array}{r}
\mu=\frac{1}{75}(\text { year })^{-1}, \gamma=\frac{1}{2}(\text { year })^{-1}, \alpha=\frac{365}{5}(\text { year })^{-1} \\
\delta=1(\text { year })^{-1}, \sigma=0.078, \beta=146(\text { year })^{-1}
\end{array}
$$

they have been identified by using clinical observations and genetic studies. The other two parameters $\rho_{1}$ and $\rho_{2}$ are fixed equal to $\rho_{1}=2$ and $\rho_{2}=2$, see [3] for some considerations about this choice. The initial conditions chosen are:

$S(0)=0.88, \quad I(0)=0.02, \quad R(0)=0.05, \quad C(0)=0.05$

The equilibrium points for the given values of parameters are:

$$
\begin{gathered}
X_{e}^{1}=\left(\begin{array}{llll}
1 & 0 & 0 & 0
\end{array}\right)^{T} \\
X_{2}^{e}=\left(\begin{array}{lllll}
0.48 & 0.0021 & 0.24 & 0.28
\end{array}\right)^{T}
\end{gathered}
$$

The first one is characterized by no infected subjects and therefore no influenza exists; computing the matrices (10) and (11) for the equilibrium point $X_{1}^{e}$, local instability and non controllability with respect to the control $u_{2}$ can be proved. In the equilibrium point $X_{2}^{e}$ the percentage of infected subjects is less than $\frac{1}{100}$ of the susceptible one; the expressions (10) and (11) become: 


$$
\begin{gathered}
A_{2}=\left(\begin{array}{cccc}
-0.32 & -69.77 & 0 & 0.5 \\
0.31 & 0 & 0 & 0.02 \\
0 & 111.35 & -1.01 & 0.29 \\
0 & -41.49 & 1 & -0.46
\end{array}\right) \\
B_{2}=\left(\begin{array}{cc}
-0.95 & 0 \\
0 & -0.0043 \\
0.95 & 0.0043 \\
0 & 0
\end{array}\right)
\end{gathered}
$$

with the eigenvalues of $A_{2}$ equal to:

$$
0.18, \quad-1.77, \quad-0.10+4.34 j, \quad-0.10-4.34 j,
$$

which implies the local instability. The controllability property for $\left(A_{2} B_{2}\right)$ is satisfied, making possible the application of the LQ regulator theory. Thus the procedure is performed referring to $X_{2}^{e}$. The weights of the matrices $Q$ and $R$ account for different strategies; as a first choice:

$$
q_{1}=q_{2}=0.1 \quad r_{1}=r_{2}=10^{-3}
$$

are assumed. This implies that in the cost index the susceptible subjects are weighted less than the infected ones; it means that the main goal is the minimization of the infected patients. The same control effort is allowed both in the preventions and in the medication. In (27) the relative weight between the state and the control penalties is assumed significantly different, both to stress the main goal of minimizing the patients, and to take into account that the state variables are normalized, whereas no constraints are introduced on the control ones. The algebraic Riccati solution $P$ of (14) and the value $g_{r}$ in (16) are:

$$
\begin{gathered}
P=\left(\begin{array}{cccc}
0.04 & -0.05 & 0.03 & 0.04 \\
-0.05 & 2.55 & -0.06 & -0.07 \\
0.03 & -0.06 & 0.04 & 0.04 \\
0.04 & -0.07 & 0.04 & 0.05
\end{array}\right) \\
g_{r}=\left(\begin{array}{c}
-0.01 \\
0.14 \\
-0.01 \\
-0.01
\end{array}\right)
\end{gathered}
$$

The optimal feedback law (15) is:

$U^{o}(t)=\left(\begin{array}{cccc}6.47 & 8.66 & -3.60 & -3.70 \\ -0.36 & 11.18 & -0.42 & -0.47\end{array}\right) \tilde{X}^{o}(t)+\left(\begin{array}{c}0.11 \\ -0.64\end{array}\right)$

Note that the eigenvalues of the dynamical matrix of the linear controlled system are:

$$
-5.61 ; \quad-0.18 ; \quad-2.78 ; \quad-2.89
$$

thus yielding the stabilizing effect on the linearized system. The state feedback (28), modified as in (19), is applied to the nonlinear original system (1)-(4), obtaining a control action not depending on the solution of the costate equations, [5]. The behaviours of the state functions and of the control actions are shown for a period of 12 months in Figures 3 and 4, respectively. The peak of the influence occurs at about 5 days from the beginning of the analysis and involves less than $16 \%$ of the population. Figure 5 shows a stronger control effort on the infected patients (control $u_{2}$ ) than for the susceptible ones. In order to study the effect of the local approximation procedure, the evolutions $S(t)$ and $I(t)$ under control (19) are compared considering both nonlinear and linearized dynamics. Figures 5 and 6 show the results for a period or 36 months. From these figures it is evident that the transient for the first year are quite different; however, in this case the nonlinearities contribute to make the controlled system converge more rapidly to the reference value.

To study the effects of the weight parameters in (13), some quantities are evaluated:

- the peak of the influence: it is evaluated as the maximum percentage of subjects that have caught the disease;

- the time of occurrence of the maximum peak: it is evaluated as the number of days from the beginning of the influenza to the day of the maximum peak of the number of infected patients $I(t)$; it is useful for the estimation of the rate of propagation of the influenza;

- the effort $E_{1}$ and $E_{2}$ of the control $u_{1}$ and $u_{2}$ respectively, evaluated as the integral of the squared control in the entire simulation period.

They are computed for different values of $q_{i}$, as in Table I, assumed fixed the values of $r_{i} i=1,2$ as in (25), and for different values of $r_{i} i=1,2$, as in Table II, assuming fixed $q_{i}$, again as in 25 . The decimal term in the evaluations of the day of the peak are reported for completeness.

In case of free evolution, reported only in Table I, the maximum percentage of infected patients is almost the $18 \%$ of the population and the peak of influenza occurs about one week after its beginning.

The most significant decrease of the peak of influence, about $13 \%$ of the total population, is obtained with a very low weight of the control $u_{2}$, at the expense of a corresponding high energy consumption $E_{2}$ and almost negligible $E_{1}$ (Table II). A good result, not as expensive as the previous one, is obtained increasing the weight $q_{2}=10$ (Table II), or when weighting very little the control parameter $r_{1}=10^{-4}$, (Table II). The reference situation of Figures 3 and 4 is shown in Table II, first row; the strategy obtained costs more from the medication point of view with respect to the prevention.

If one tries to minimize essentially the infected subjects and almost disregards the minimization of the susceptible ones (Table I, first row) the result is not so satisfactory, since the peak of the influence is almost $17 \%$; this confirms the reason of the choice of the cost index (9).

The results of the proposed approach appear rather satisfactory since the closed form solution shows a significant reduction of the influenza disease.

In this paper the information about the number of susceptible and infected subjects has been considered as known; an analogous problem could be faced assuming this knowledge not available, being known only the number of recovered subjects $R$; in this realistic case an observer is required, once it is verified the observability of the couple $\left(A_{2} C\right)$. For the 
proposed choice of parameters, it could be easily verified the non singularity of the controllability matrix:

$$
O=\left(\begin{array}{c}
C \\
C A_{2} \\
C A_{2}^{2} \\
C A_{2}^{3}
\end{array}\right)=\left(\begin{array}{cccc}
0 & 0 & 1 & 0 \\
0 & 111.35 & -1 & 2.9 \\
34.5 & -124.4 & 1.31 & 1.8 \\
-49.6 & -2337.1 & 0.48 & 14.32
\end{array}\right)
$$

Therefore the control law should be applied with the estimated state, thus requiring the separated determination of the gain of the observer, and of the linear quadratic tracking controller, (28).

To complete the study, a comparison between the approach and the results obtained in this paper and the optimal control proposed in [3] is now discussed, being the latter the most similar, in terms of the modelling and of the optimal control approach. Nevertheless, differences are present; in that paper the cost index was quadratic only in the control, aiming at minimizing the number of susceptible and of the infected subjects and constraints on the control actions were assumed.

The direct application of the Pontryagin minimum principle allowed to obtain, only numerically, the optimal control.

Various models describing influenza disease can be found in literature, presenting slight variations according to the peculiar aspects to be analysed; classical optimal control design techniques are adopted as in [9], where again the solution must be computed numerically.

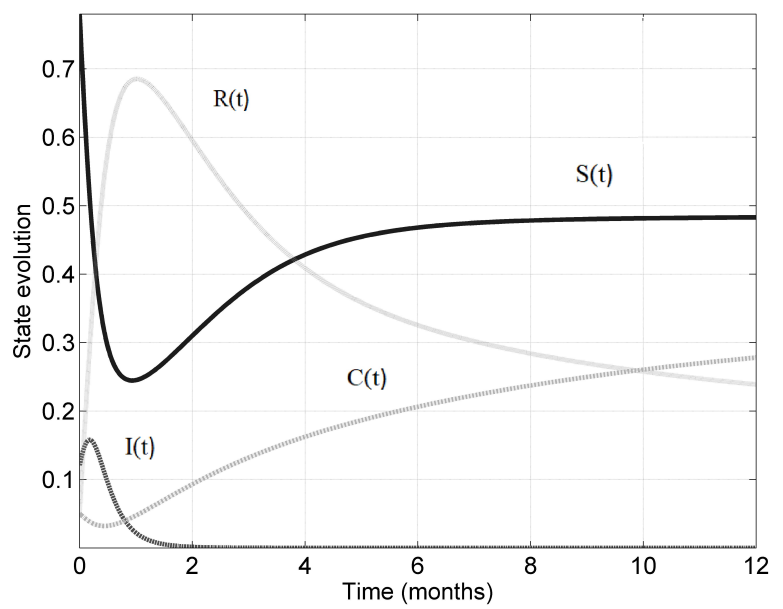

Fig. 3. State evolution with the proposed control actions

TABLE I

COMPARISON

\begin{tabular}{l|c|c|c|c}
$\left(q_{1} ; q_{2}\right)$ & Peak of the influence & Day & $E_{1}$ & $E_{2}$ \\
& & & & \\
\hline$(0.0055 ; 1)$ & 0.167 & 6.12 & 0.84 & 2.19 \\
\hline$(0.01 ; 1)$ & 0.166 & 6.12 & 1.34 & 3.24 \\
\hline$(0.1 ; 10)$ & 0.143 & 3.60 & 437.44 & 75.22 \\
\hline Free evolution & 0.179 & 6.90 & & \\
\hline \hline
\end{tabular}

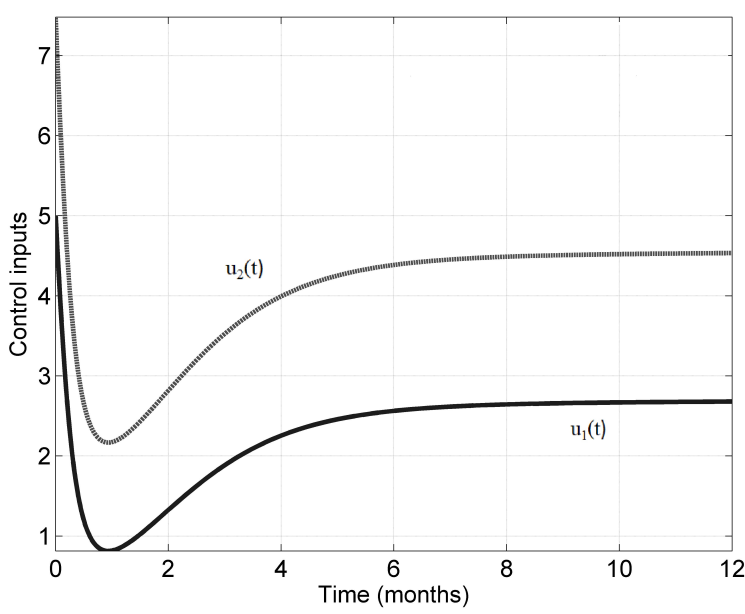

Fig. 4. Control actions both on susceptible and infected subjects

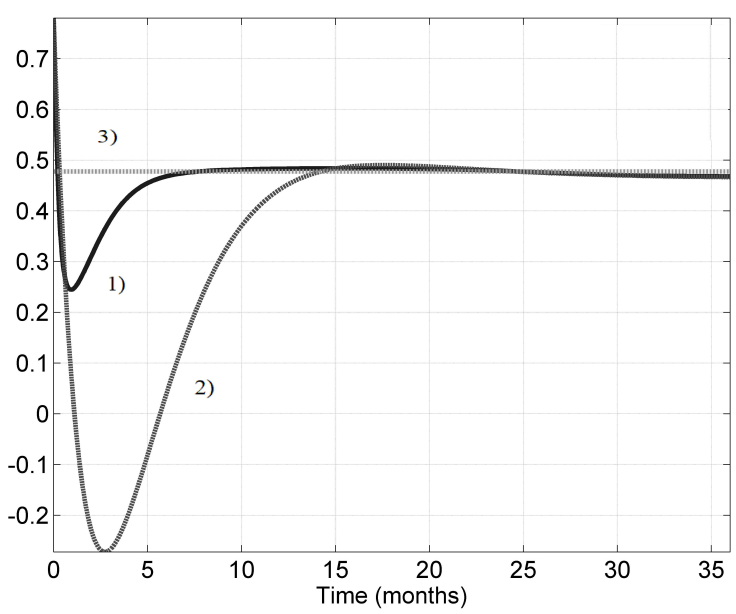

Fig. 5. Comparison of the behaviour of the percentage of susceptible subjects of the controlled non-linear model with the linearized one: 1) Nonlinear dynamics; 2) Linear dynamics; 3) equilibrium value $X_{2}^{e}(1)$

TABLE II

COMPARISON

\begin{tabular}{l|c|c|c|c}
$\left(r_{1} ; r_{2}\right)$ & Peak of the influence & Day & $E_{1}$ & $E_{2}$ \\
\hline$\left(10^{-3} ; 10^{-3}\right)$ & 0.158 & 5.25 & 5.95 & 24.89 \\
\hline$\left(10^{-4} ; 10^{-3}\right)$ & 0.144 & 3.55 & 423.81 & 5.76 \\
\hline$\left(10^{-3} ; 10^{-4}\right)$ & 0.136 & 3.82 & 0.002 & 1366.56 \\
\hline \hline
\end{tabular}

\section{CONCLUSIONS}

In this paper, the SIRC influenza epidemic model with the four compartments of susceptible, infected, removed and cross immune subjects is considered. It well describes the possibility of a partial immunization in an epidemic disease; therefore, a removed subject, after a period, could get ill by different strain of the same viral subtype.

The problem of controlling such an epidemic disease has 


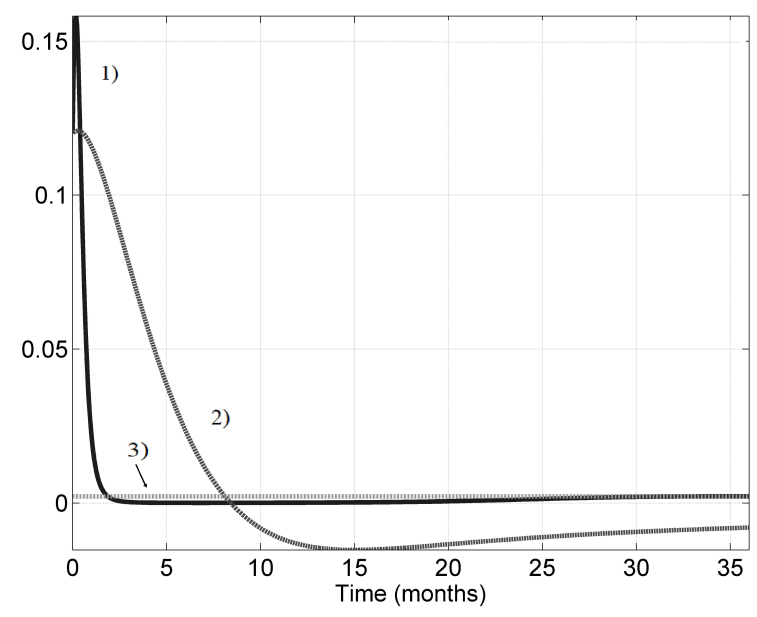

Fig. 6. Comparison of the behaviour of the percentage of infected subjects of the controlled non-linear model with the linearized one: 1) Nonlinear dynamics; 2) Linear dynamics; 3) equilibrium value $X_{2}^{e}(2)$

been tackle in the framework of linear quadratic regulator after a linearization of the original nonlinear model; this control action has been applied on the original nonlinear system with the advantages of having a control depending on the current state.

The numerical results show the effectiveness of the approach, especially with respect to the severity of the illness measured in terms of lower peak of the influenza and a faster decrease of the total number of infected subjects, when compared with the absence of control actions. With respect to analogous optimization procedures, the main advantage relies in the feedback control structure, with at least comparable numerical results; this control structure also allows the use of a state estimator when all the information is not available.

In further developments, effort will be devoted to determine a suitable tuning of the weighting matrices and to consider real data of influenza-like disease.

\section{REFERENCES}

[1] H. Behncke, "Optimal control of deterministic epidemics," Optimal control applications and methods, vol. 21, 2000.

[2] P. Di Giamberardino and D. Iacoviello, "Optimal control of SIR epidemic model with state dependent switching cost index," Biomedical Signal Processing and Control, vol. 31, 2017.

[3] D. Iacoviello and N. Stasio, "Optimal control for SIRC epidemic outbreak," Computer Methods and Programs in Biomedicine, 2013.

[4] P. Di Giamberardino, L. Compagnucci, C. D. Giorgi, and D. Iacoviello, "Modeling the effects of prevention and early diagnosis on HIV/AIDS infection diffusion," IEEE Transactions on Systems, Man and Cybernetics: Systems, 2018

[5] P. Di Giamberardino and D. Iacoviello, "LQ control design for the containment of the HIV/AIDS diffusion," Control Engineering Practice, vol. $77,2018$.

[6] C. Nowzari, V. M. Preciado, and G. J. Pappas, "Analysis and control of epidemics. a survey of spreading processes on complex networks," IEEE Control Systems Magazine, 2016.

[7] R. Casagrandi, L. Bolzoni, S. Levin, and V. Andreasen, "The SIRC model and influenza," A. Mathematical Biosciences, vol. 200, 2006.

[8] A. Zeb, G. Zaman, M. Chohan, S. Momani, and V. Ertur, "Analytic numeric solution for SIRC epidemic model in fractional order," Asian Journal of Mathematics and Applications, 2011.
[9] J. Lee and H. Kwon, "Optimal control of an influenza model with seasonal forcing and age-dependent transmission rates," Journal of Theoretical Biology, vol. 317, 2013.

[10] M. El-Shahed and A. Alsaedi, "The fractional SIRC model and influenza a," Mathematical problems in Engineering, 2011.

[11] E. Bakare, A. Nwagwo, and E. Danso-Addo, "Optimal control analyis of an SIR epidemic model with constant recruitment," International Journal of Applied Mathematical Research, vol. 3, 2014.

[12] A. Lashari, "Optimal control of an sor epidemic model with saturated treatment," Applied Math.Inf.Science, vol. 10, 2016.

[13] P. Di Giamberardino and D. Iacoviello, "Modeling and control of an epidemic disease under possible complication," Proceedings of the 22nd International Conference on System Theory, Control and Computing, pp. 67-72, 2018

[14] L.Pang, S.Ruan, S.Liu, Z.Zhao, and X.Zhang, "Transmission dynamics and optimal control of measles epidemics," Applied mathematics and computations, vol. 256, pp. 131-147, 2015.

[15] D. Iacoviello and G. Liuzzi, "Fixed/free final time SIR epidemic models with multiple controls," International Journal of Simulation and Modelling, vol. 7, no. 2, pp. 81-92, 2008

[16] P. Di Giamberardino and D. Iacoviello, "Analysis, simulations and control of a new measles epidemic model," International Conference on Informatics in Control, Automation and Robotics, p. In press, 2019.

[17] H. Chang and A. Astolfi, "Control of HIV infection dynamics," IEEE Control Systems, 2009.

[18] H. Joshi, "Optimal control of an HIV immunology model," Optimal control applications and methods, vol. 23, 2002.

[19] Y. Zhou, K. Yang, K. Zhou, and C. Wang, "Optimal treatment strategies for HIV with antibody response," Journal of applied mathematics, 2014

[20] P. Di Giamberardino and D. Iacoviello, "Optimal control to reduce the HIV/AIDS spread," Proceedings of the 22nd International Conference on System Theory, Control and Computing, 2018.

[21] P. Di Giamberardino and D. Iacoviello, "HIV infection control: A constructive algorithm for a state-based switching control," Int. Journal of Control, Automation and Systems, vol. 16, no. 3, pp. 1469-1473, 2018.

[22] S.Ahmad and M. Tokhi, "Linear quadratic regulator (LQR) approach for lifting and stabilizing of two-wheeled wheelchair," 4st International Conference on Mechatronics, 2011.

[23] C. Rodrigues, R. Kuiava, and R. Ramos, "Design of a linear quadratic regulator for nonlinear systems modeled via norm-bounded linear differential inclusions," 18st IFAC World Conference, 2011.

[24] W. Li and E. Todorov, "Iterative linear quadratic regulator design for nonlinear biological movement system," 1st International Conference on Informatics in Control, Automation and Robotics, 2017.

[25] S. Eshghi, M. Khouzani, S. Sarkar, and S. Venkates, "Optimal patching in clustered malware epidemics," IEEE Transactions on Networking, vol. 24, 2016.

[26] M. Bloem, T. Alpcan, and T. Basar, "Optimal and robust epidemic response for multiple networks," Proc. Of the 46th IEEE Conference on Decision and Control, 2007. 